Inkyung Choi — University of Wisconsin-Milwaukee

\title{
Visualizations of Cross-cultural Bibliographic Classification: Comparative Studies of the Korean Decimal Classification and the Dewey Decimal Classification.
}

\begin{abstract}
The changes in KO systems induced by sociocultural influences may include those in both classificatory principles and cultural features. The proposed study will examine the Korean Decimal Classification (KDC)'s adaptation of the Dewey Decimal Classification (DDC) by comparing the two systems. This case manifests the sociocultural influences on KOSs in a cross-cultural context. Therefore, the study aims at an indepth investigation of sociocultural influences by situating a KOS in a cross-cultural environment and examining the dynamics between two classification systems designed to organize information resources in two distinct sociocultural contexts. As a preceding stage of the comparison, the analysis was conducted on the changes that result from the meeting of different sociocultural feature in a descriptive method. The analysis aims to identify variations between the two schemes in comparison of the knowledge structures of the two classifications, in terms of the quantity of class numbers that represent concepts and their relationships in each of the individual main classes. The most effective analytic strategy to show the patterns of the comparison was visualizations of similarities and differences between the two systems. Increasing or decreasing tendencies in the class through various editions were analyzed. Comparing the compositions of the main classes and distributions of concepts in the KDC and DDC discloses the differences in their knowledge structures empirically. This phase of quantitative analysis and visualizing techniques generates empirical evidence leading to interpretation.
\end{abstract}

\subsection{Problems statement}

As advances in information communication and technology (ICT) break national, social, and cultural boundaries, use of bibliographic classification systems also crosses social and cultural borders. Countries other than those in North America, for instance, have adopted the Dewey Decimal Classification (DDC) for organizing their library collections. In a trend of globalization, however, the question of proper localization of information systems beyond translation or assimilation is still in dispute. In reaction to globalization, indigenization of interoperable information systems is actively discussed (Leidner 2010; Doyle 2006).

Because classification is socially constructed, it carries its own assumptions about the world and may have significant consequences not only for the knowledge user but also for society. Recognizing these sociocultural influences, Knowledge Organization $(\mathrm{KO})$ research has examined how multiple sociocultural viewpoints are realized in KOSs. Along with the notion of cultural warrant (Beghtol 2002, 45), sociocultural influences have received attention through discoveries of categories and/or their relationships in Knowledge Organization Systems (KOSs) that result from social and cultural factors. Applying empirical and interpretative methods such as 
tracing changes to the composition of a KOS, those KO studies mostly aim to reveal the dynamics and evolution of knowledge structures from the sociocultural changes in one society or one culture (Salah et al. 2012; Tennis 2012). Such studies also explore multiple perspectives in organizing knowledge derived from diverse sociocultural contexts.

Given increasing cross-cultural use of classification, it is no longer true that current classifications exist for only one society or one culture. Thus, it is crucial that the existing classification systems ethically respect the embedded cultures, which calls for a survey of different cultures. However, few KO studies have illustrated how different cultures are reconciled through conflicts and harmonization within a KO structure beyond pointing out the need to recognize and identify the sociocultural perspectives.

The changes in KOSs induced by sociocultural influences may include those in both classificatory principles and cultural features. The proposed study examines the Korean Decimal Classification (KDC)'s adaptation of the DDC by comparing the two systems. This case manifests the sociocultural influences on KOSs in a crosscultural context. Therefore, the study aims at an investigation of sociocultural influences by situating a KOS in a cross-cultural environment and examining the dynamics of two classification systems designed to organize information resources in two distinct sociocultural contexts.

\subsection{Backgrounds}

This section illustrates metatheoretical assumptions of the current study through literature in KO field. Metatheoretical assumptions are more like philosophical views, thus more general than theories (Hjørland 1998). They are the assumptions made to generate specific theories and often recognized in the elaboration of the concepts by use of certain terms in texts. In this study, I will discuss a notion of 'intercultural warrant' as a methodological suggestion followed by metatheoretical assumptions regarding the impact of sociocultural influences on KOS. This section discusses fundamental concepts related to intercultural warrant. And I introduce the previous studies of the KDC regarding the DDC's influences.

\subsection{Metatheoretical assumptions}

\subsubsection{Contextual bound knowledge organization}

In the literature of $\mathrm{KO}$, there were discussions of whether knowledge is bound to contexts. The past KO studies have recognized two philosophical schools of thoughts underpinning the development of knowledge organization principles: ontology and epistemology. The recent studies of KO tend to embrace epistemological views, which bring scholarly attentions to contexts that shape meanings and forms of knowledge. According to Mai (2010), there have been evident shifts "from classification-as- 
ontology, in which everything is defined as it is, to a more contemporary notion of classification-as-epistemology, in which everything is interpreted as it could be (711)". Along with the increasing interest in epistemology, the importance of context in organizing knowledge and information retrieval has been discussed. Olson (2009) explores the historical aspects of theoretical foundations for classification at various times and noted that no neutral classification exists through the historical transitions of classifications. According to her, Sayers (1926) and Bliss (1929) implies that even natural classification adhering to the natural order (ontology) must overlap with purposive classification (epistemology) in some ways.

Regarding the advanced information technology which brought explosive amounts of information, use of information in practice also have focused technical developments which can be largely seen in two main streams. They are improving the efficiency and efficacy of KOSs and understanding of social, political, and cultural influences on KOS.

Hjørland (2012) and Smiraglia (2014) examine the challenges facing KO in the Internet era and the value of efforts to catalog and classify "recorded knowledge." It might seem that there is no need for KO or even Library and Information Science (LIS), given many search engines that make it possible to search for and access information with a few keywords or mouse clicks. Searching for information is no longer the exclusive domain of traditional information services such as libraries. Knowledge as something that constitutes decision making (Lester and Wallace 2007) - acquiring information is not enough to make a person knowledgeable. Fulfillment of the need for knowledge could begin in a search for information, but knowledge can only be obtained through a synthesis of information. To synthesize information, we need contexts from which information can be found. Mere information retrieval is not what satisfies users' needs, nor does it replace what libraries contribute to society. Through such understanding of philosophical views of knowledge and technical developments in organizing knowledge, this study posits that KOSs are contextually bound. Especially, KOS are shaped by social and cultural influences.

\subsubsection{Social and cultural influences in organizing knowledge.}

The distinction between the ontological and the epistemological approaches in KO could shed light on KO's foundation regarding social and cultural conditions beyond technological advances. Many KO scholars investigate epistemology of a certain domain, with special attention to social and cultural aspects of organizing knowledge, to construct valid KOSs. Hjørland $(2008,16)$, for example, advocates for the domain analytic approach to $\mathrm{KO}$ and states that "domain analysis is a sociologicalepistemological standpoint". This approach represents current epistemological thought and simultaneously gives room for social and cultural viewpoints to interplay in KO. 
In the form of conceptual study, they are shedding light on the importance of epistemology and contextuality of KOSs. Since KO, as a discipline has been developed upon the strong foundation of not only theory but also practice, it is necessary to find what the acknowledgment of social influences in KO brings to bear on practice as well. Classification research has applied various approaches to examining meanings through empirical analysis of text-based data derived from classification. KO scholars such as Tennis (2006, 2007), Olson (2001), and Fox (2013) have examined changes to a certain range of topics represented in classifications through textual and structural analysis of the concepts. These studies tested how a certain concept has been changed and shifted in KOSs. The analysis is to track the changing concepts in continuous modifications of a particular KOS. Thus, vertical and historical traces examining changes over time, as well as synchronic traces revealing boundary of the concept, occur in the analysis. Following the conceptual changes of a particular KOS proved empirically the social influences in organizing knowledge, implying methodological directions in classification research.

Since international collaborations in KO filed make classification systems across cultures available to KO scholars, the attempts to compare classifications of distinct cultures start to occur. The presuppositions, first, are derived from an acceptance of multiple cultures shaping different forms of knowledge and knowledge organizations. That multiplicity helps to expose our own limits and limitations, and comparative approaches to knowledge make the previously unthinkable or the unthought in some ways thinkable by uncovering those limits. The crucial point here is that remotely different cultures have widely different conceptual schemes and systems of classification, each of which may effectively make the other culture acutely aware of its own historical and contingent nature (Xie, 2011). Taking this comparative mind, the studies investigate the epistemologies of non-western or ancient knowledge structures, with implications for the current KOS (Neelameghan and Raghavan 2012; Lee 2012)

\subsection{Previous studies of the KDC in comparison of the DDC}

As the KDC is currently a national library classification of South Korea, there are general texts introducing various aspects of the system (Oh, Bae, and Yeo 2002; 2009). Recent Korean works analyzing the KDC address more functional and systemic issues, such as evaluating or updating classificatory structures and coverage for subject areas (Kim 2009; Yeo, Lee, and Oh 2008; Yeo, Park, Hwang, and Oh 2008; Oh, Bae, and Yeo 2008). In evaluating and making suggestions for the desired direction of the KDC changes, the authors also compare the KDC with the DDC or the NDC. Their comparisons, however, mostly consider structural problems within the $\mathrm{KDC}$, without sociocultural concerns. 
In one analysis (Oh 2012), some parts of the KDC, such as schedules of main classes or notations, were compared with the DDC to see 1) the influences of the DDC on the KDC, and 2) the unique characteristic of the KDC. Although the comparison was not designed for comprehensive analysis of sociocultural differences between the KDC and the DDC, it shows the possibility of comparison as an analytical tool for studying sociocultural issues in an adaptation of classification. Kwasnik \& Chun (2004) also conducted another comparative study of the KDC and the DDC by investigating both semantic contents and structures of the two classifications. This study offers the framework for comparing two different knowledge structures. However, their findings are limited to only some observed phenomena from intentionally selected parts of the KDC, not leading to contemplation of sociocultural factors of South Korea and North America. The current study attempts to capture knowledge structures of two systems taking bottom-up approaches and to focus the adaptation efforts analyzing the conflicts and harmonization of two distinct cultures.

\subsection{Research questions}

The KDC was independently developed as the Korean national classification using the basis of the DDC principles, so Korean culture appears in the KDC as a proper reflection of the cultural warrant. At the same time, the KDC also inherits some cultural features of the DDC. In the KDC's adaptation of the DDC, it either aligns with the DDC or reflects specific aspects of Korean culture. These interactions between the two KOSs and inherited cultural features call for an inquiry into theory explaining complex cultural warrants in cross-cultural environments. Thus, this study will examine the adaptation of a popular classification to accommodate a local culture when two different cultural warrants merge into an intercultural warrant. The study, therefore, will answer the following question: "What are the changes from the KDC's adaptation of the DDC in view of its intercultural warrants?"

\subsection{Methods}

Classification research has applied various approaches of content analysis to examining meanings through empirical analysis of text-based data derived from classification. KO scholars have examined classifications in unique ways regarding its features attributing to concept theory. Smiraglia, Scharnhorst, Salah, and Gao (2013) suggested that the application of a quantitative approach and visualization to classification research permits observation of changes in classification such as size, composition, growth, and distribution. Thus, comparing the compositions of the main classes and distributions of concepts in the KDC and DDC disclosed the differences in their knowledge structures empirically. Furthermore, I examined the degrees and patterns of variation to see the main classes that present the most different compositions and distributions.

In a decimal classification system, each class represents a broad discipline. 
Although the KDC and the DDC order their classes slightly differently, the ten classes representing broad disciplines do match (Table1). Thus, this study specifically looks for variation within each of the ten matching main classes between the two classifications. Doing so, it assumes: the KDC's adaptation to meet its sociocultural needs is observable in its changes of classification numbers from the DDC within individual main classes. To compare the knowledge categories and structures of the KDC and the DDC, I collected and compared all classification numbers from a total of 6 editions in digital formats - three of the recent KDC editions and three DDC editions timely matched with the KDC editions' release. With the help from Online Computer Library Center (OCLC), the owner of the DDC, I obtained the datasets containing the electronic records representing individual DDC class numbers. Also, I could obtain the electronic data sets containing records representing individual class numbers used in a recent study of the KDC (Jeon 2015). This study first compares the three recent editions of the DDC and the three recent editions of the KDC and later mainly compared DDC23and KDC6 for the classes showing the most difference.

\begin{tabular}{|l|l|l|}
\hline DDC 23 & Class & Subjects \\
\hline Subjects & 000 & $\begin{array}{l}\text { General works (\& Computer science and } \\
\text { Information) }\end{array}$ \\
\hline $\begin{array}{l}\text { General works, Computer science and } \\
\text { Information }\end{array}$ & 100 & Philosophy (\& psychology) \\
\hline Philosophy and psychology & 200 & Religion \\
\hline Religion & 300 & Social sciences \\
\hline Social sciences & 400 & Natural sciences \\
\hline Language & 500 & Technology \& engineering \\
\hline Pure Science & 600 & Arts (\& recreation) \\
\hline Technology & 700 & Language \\
\hline Arts \& recreation & 800 & Literature \\
\hline Literature & 900 & History (\& geography) \\
\hline History \& geography & \multicolumn{2}{|c|}{ Table 1.10 main classes of the DDC and the KDC }
\end{tabular}

The Language of the data sets for the KDC editions is Korean, so I referred English captions provided in the paper copies of the KDC editions. This comparative analysis was conducted on the changes that result from the meeting of different sociocultural feature in a descriptive method, aiming to identify variations between the two schemes in comparison of the knowledge structures of the two classifications, in terms of the quantity of class numbers that represent concepts and their relationships in each of the individual main classes. The most effective analytic strategy to show the hidden patterns of the compared classifications was visualizations of similarities and differences between the two systems. All class numbers including three-digit integer and combined auxiliary numbers were used for this analysis. Increasing or decreasing tendencies in classes through various editions were analyzed. XML editors, MS excel, 
and $\mathrm{R}$ were used for data formatting and $\mathrm{R}$ (ggplot2 packages), Tableau, and MS Excel were also used for graphical visualizations of the data.

\subsection{Results}

As the order of the KDC main classes is partially different than that of the DDC, I match them first by disciplines and then compare topics within each individual class. Figure 1 shows the counts of classification numbers by disciplines representing the differences of DDC23 and KDC6 in a distribution of subjects. Large differences in Technology \& Science and Social Science are noticeable.

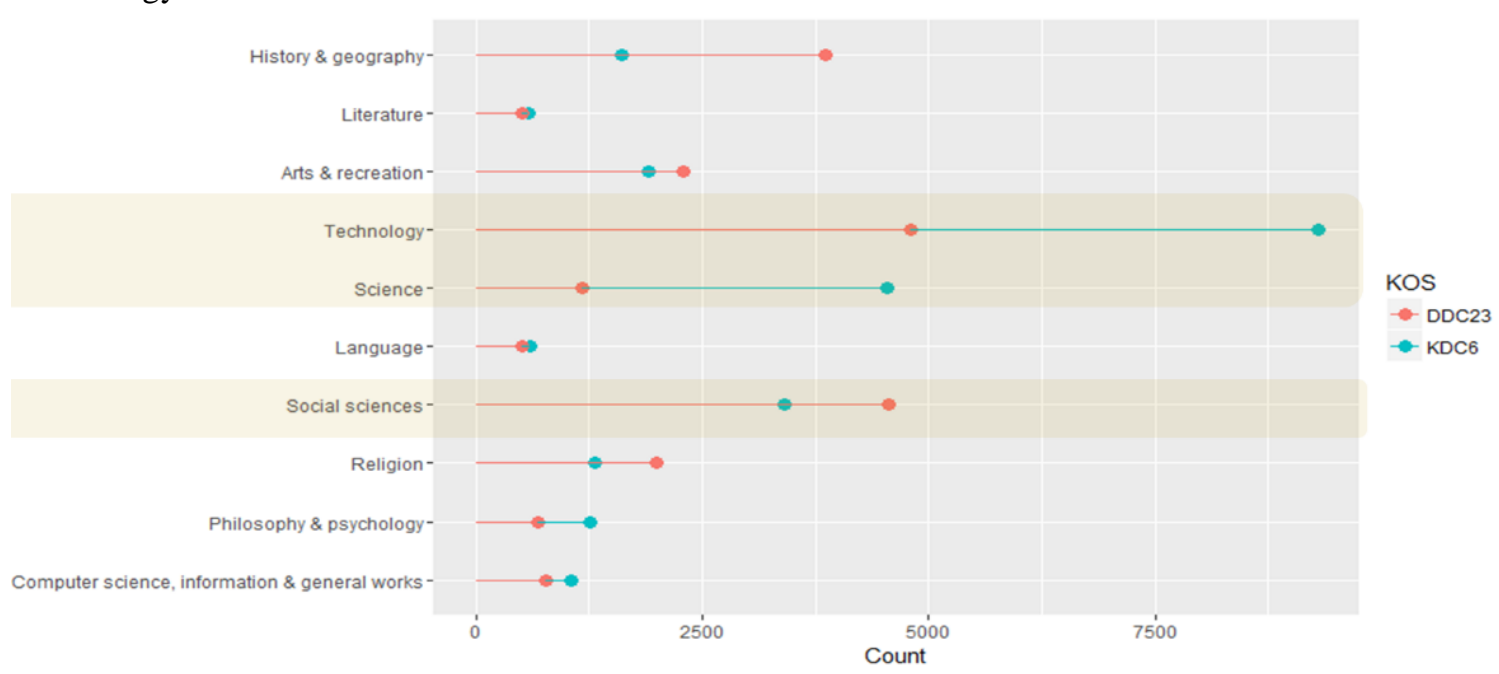

Figure 1. Distributions of class numbers of the DDC 23 and the KDC 6 by disciplines matched

The recent three KDC editions also show higher percentages of class numbers in the main class of Technology (the KDC4 -36.78\%, the KDC5 $-36.34 \%$ and the KDC6 - 36.38\%) while the recent three DDC editions show higher percentages of the main classes for Social Science in Figure 2 (the DDC20-20.60\%, the DDC22 - 20.77\%, and the DDC23-21.52\%). The DDC editions also consistently present higher percentages of class numbers in the main class of History \& geography. Given the differences between two systems regarding the KDC as a national library classification and the DDC as a universal library classification, this study chooses the main class of social science as a case of the DDC having more class numbers over the main class of history and geography. 

studies of the Korean Decimal Classification and the Dewey Decimal Classification. NASKO, Vol. 6. pp. 39-55.

KOS
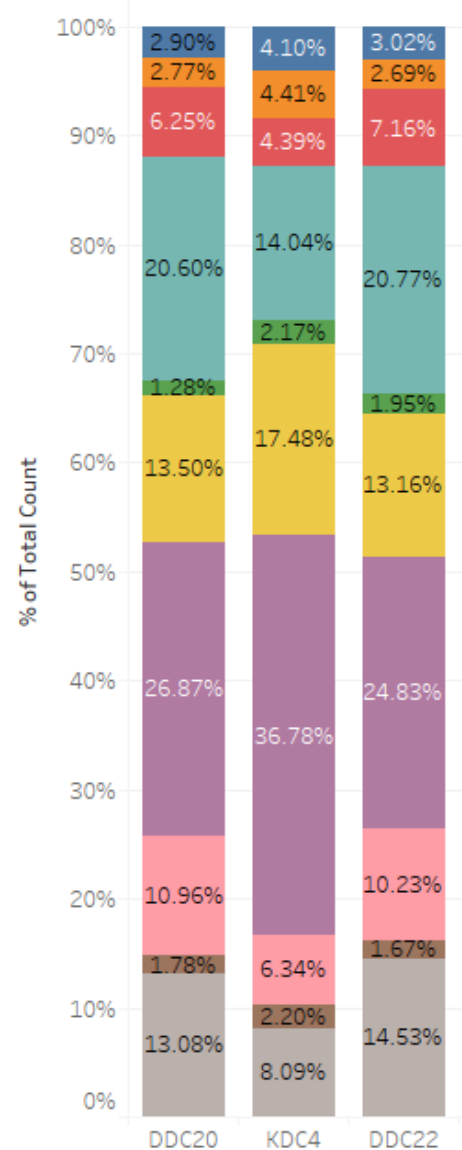
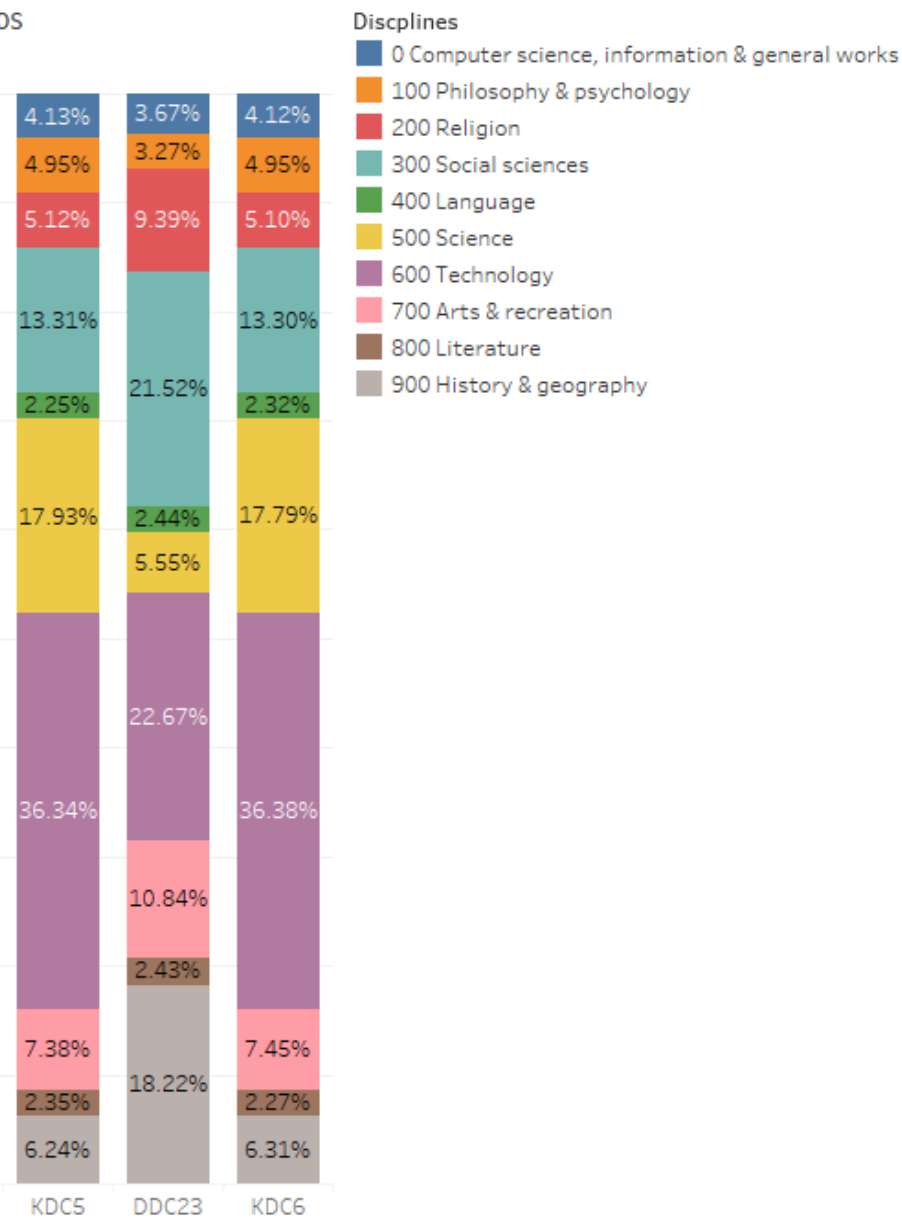

$\%$ of Total Count for each KOS. Color shows details about Discplines. The view is filtered on KOS, which keeps 6 of 6 members.

Figure 2. Percentages of main classes for the DDC 20, 22, \& 23/ the KDC 4, 5, \&6

In addition, compared to the recent three DDC editions, the recent three KDC editions do not show much change in a total quantity of class numbers (Figure 3 ). 

studies of the Korean Decimal Classification and the Dewey Decimal Classification. NASKO, Vol. 6. pp. 39-55.

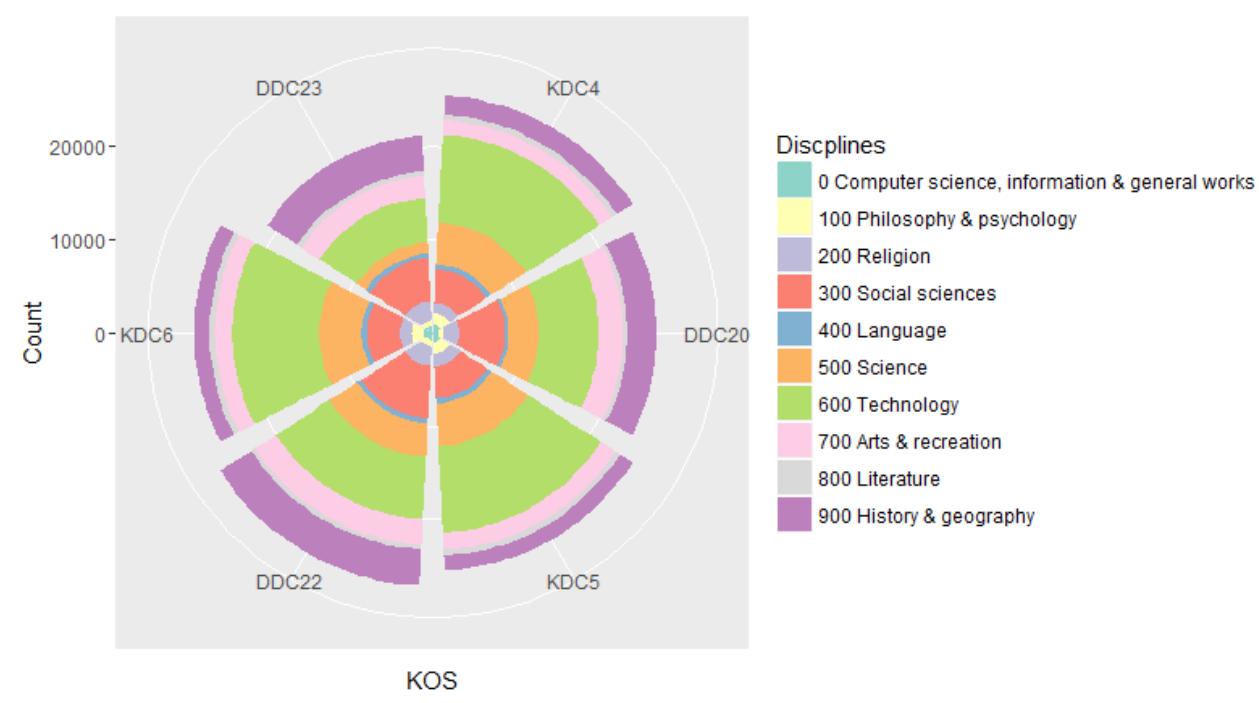

Figure 3. Distribution of main classes for the DDC 20, 22, \& 23/ the KDC 4, 5, \&6

But, there were noticeable differences in the number of records between the earlier editions of KDC - the 1st, 2nd, and 3rd - and the recent KDC editions - the 4th, 5th, and 6th, especially in the main classes of technology \& engineering (600). In tracking of the changes of the KDC editions in comparison of the DDC editions, the recent three editions of the KDC tend to reflect more dramatic changes of Korean society. 


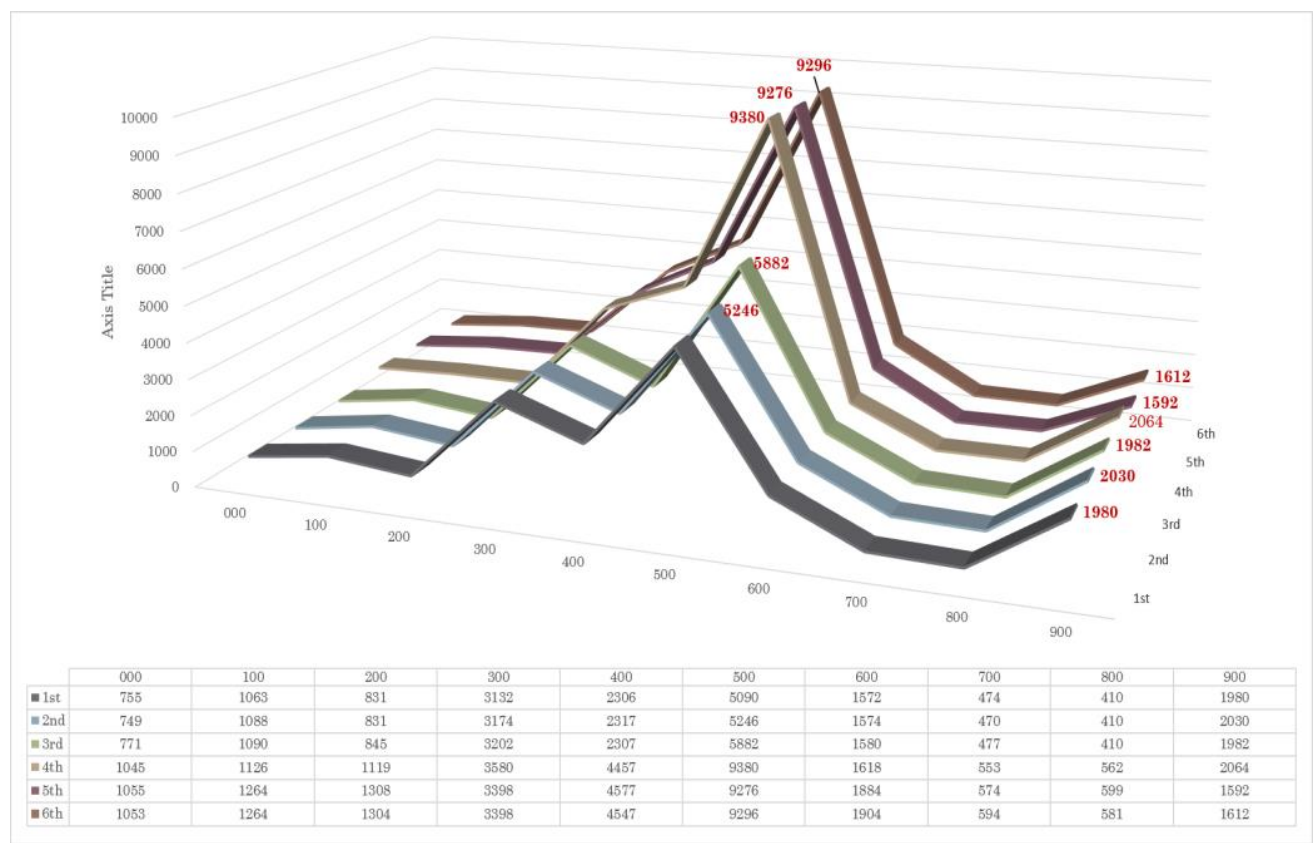

Figure 4. Chronological changes in distribution of class numbers -from the KDC1 to 6

Regarding differences in Technology/Social Science classes between DDC23 and KDC6, I compared the main classes of Social science \& Technology in the KDC6 and the counterparts in the DDC23. The figure 5 and 6 show all three-integer class numbers in each class. The colors indicate two classifications - the DDC 23 and the KDC 6. The graphs also display the counts of all class numbers combined with auxiliary numbers (e.g. 301.0820) for each three-integer class number (e.g. 301). The size of the counts and linear display of all three-integer class number show how they are distributed by sub-classes. The 10 sub-classes of two main classes, social science and technology, were integrated into 9 sub-classes in matching the sub-disciplines (e.g. Economics and Commerce in the DDC23 were integrated into one sub-class, while Public administration and military science of the KDC 6 were integrated).

Figure 5 uses class numbers 300-399 as both the DDC and the KDC designate them for the main class of social science, while Figure 6 uses 0-99 as the DDC have 600699 and the KDC have 500-599 for the main class of technology.

In Social science (300 and both in the KDC6 \& the DDC 23), except for the three sub-classes - 1. Social sciences, sociology, \& anthropology, 2. Statistics, 8.

Education, other six sub-classes were in different locations. In Technology (600 in the DDC $23 \& 500$ in the KDC6), only two sub-classes - 1. Technology \& 2. Medicine \& health were in the same order. This different order and sizes of sub-classes are expected to account for the fact that the KDC as a national library classification had to largely make adaptation of the DDC as a universal classification. 

studies of the Korean Decimal Classification and the Dewey Decimal Classification. NASKO, Vol. 6. pp. 39-55.

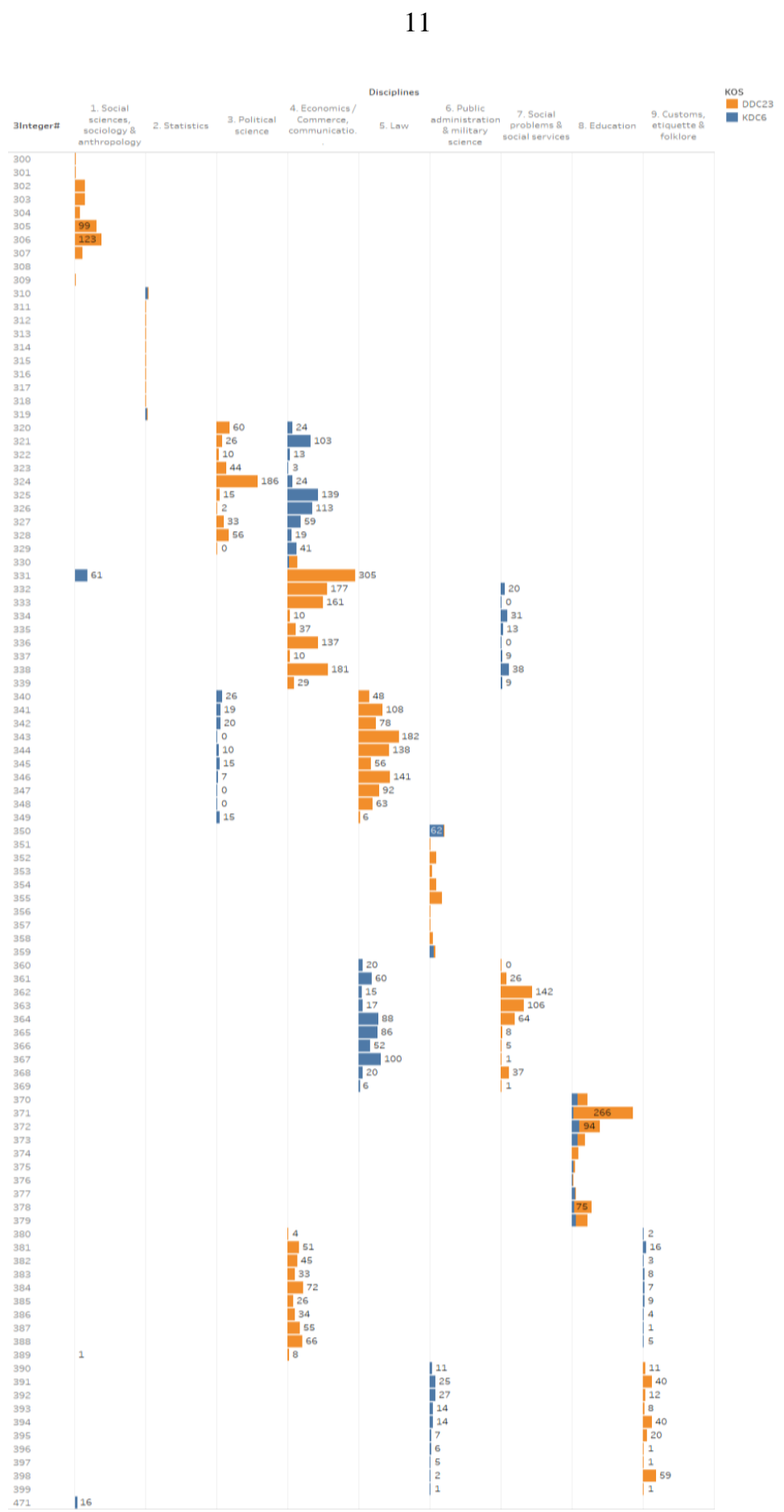

Figure 5 Distributions of sub-classes (social science) of the DDC 23 and the KDC 6 in size and location 

studies of the Korean Decimal Classification and the Dewey Decimal Classification. NASKO, Vol. 6. pp. 39-55.

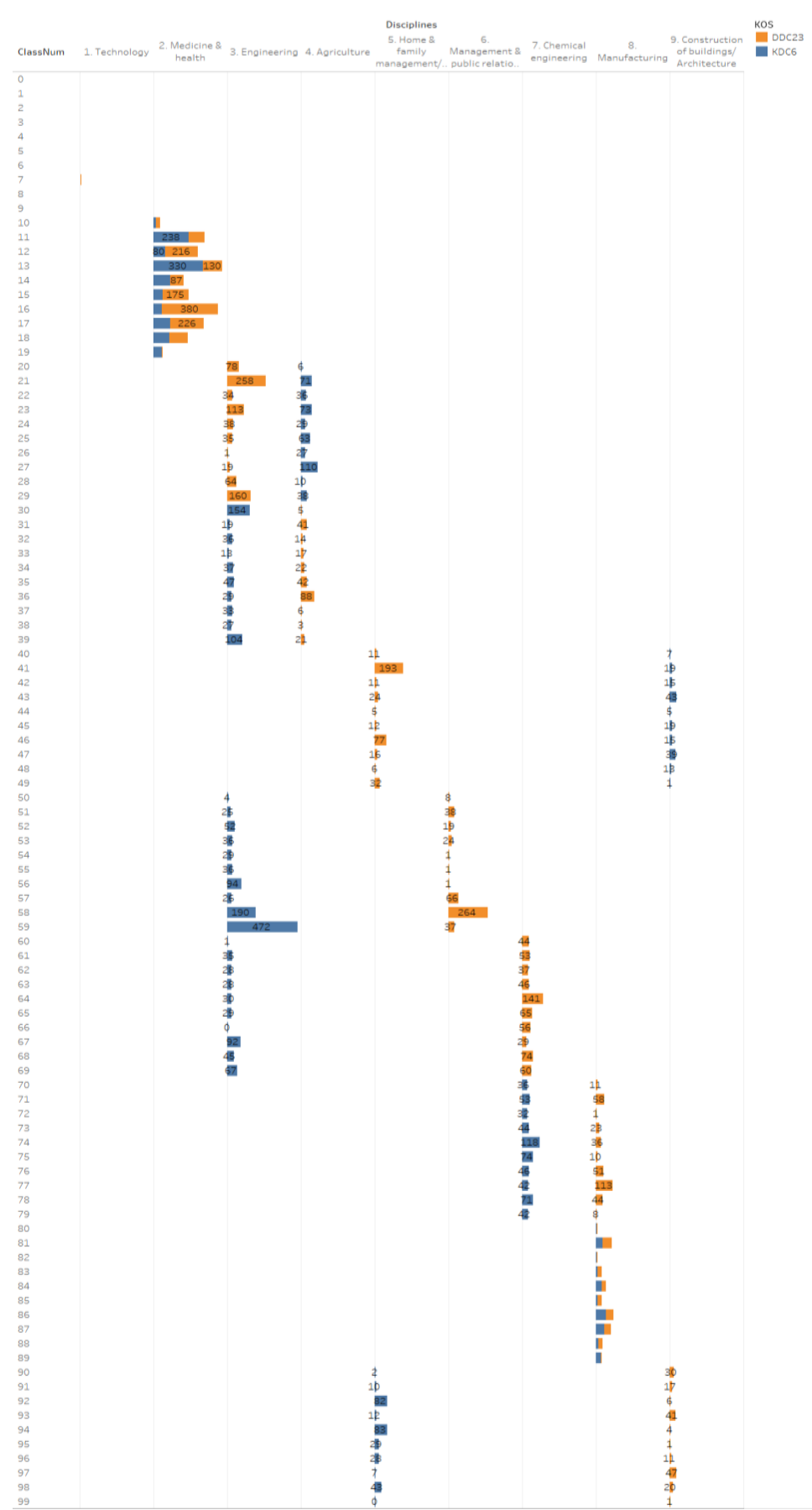

Figure 6 Distributions of sub-classes (Technology) of the DDC 23 and the KDC 6 in size and location 


\subsection{Discussions and conclusions}

\subsection{Universal or national library classification}

There is a challenge in comparison of the DDC and the KDC, given that each classification's directivity is not equal. The DDC aims to become universal library classifications such as other major library classifications such as the Library of Congress Classification (LCC) and the Universal Decimal Classification (UDC). Whereas, the KDC calmed itself to be a Korean national library classification, as the system has been developed due to limitations of the DDC in organizing Korean specific literature. Thus, this study presupposes that the universal library classification and the national library classification have different goals in comparison of the DDC and the KDC.

Interestingly, the differences of universal and national library classification are not in clear cut although two library classifications have different coverages of knowledge in their goals. The DDC has coped a vast scope of knowledge and expanded the scope through more cooperation with those countries across cultures. If applying a lens of the cultural warrant, however, it can't be denied that the scope of knowledge and cooperation are still based on North American and European countries so far. Likewise, although the KDC's development of classificatory structures is limited to a nation usage, the influence of the DDC or other universal classifications exist from the early development of the classification. Furthermore, given the tendency of Korean academic disciplines to have a huge influence of North American academic disciplines as well as international knowledge streams through academic exchanges, a scope of national library classification also can't be in a clear cut. Thus, it is understood that universal library classification and national library classification have different directivity in a scope of knowledge but the directions are gradual. Taking these gradual differences of universal and national library classifications into consideration, the cases of the DDC widely used across cultures can be divided into two categories: translation and adaptation. Both cases call for intercultural survey of national cultures and classification systems that embed those cultures

Translation of the DDC imposes the same DDC's class number so that it ensures that DDC numbers can communicate across linguistic boundaries. The DDC and the translated DDC are expandable to accommodate diverse social and cultural characteristic by respecting vernacular contents which are based on literary warrant (Beall 2003). Having the DDC translated affects the DDC to be updated for the need of diverse cultures. Adaptation of the DDC is not to have the influences over both systems. In this case, intercultural warrants emerge as in supporting multicultural characteristics. Coexisting two cultures interplay within one system. Because the KDC does not share same numeric notations, the KDC is built and modified radically based on the Korean needs. The needs are not based on literary warrant (Jeon 2015), but 
more on socio-political and user warrants. (Or I would argue user warrant reflective of cultural warrant). For example, in the early development of the KDC by the Korean National Library Associations back in 1960's (Cho 1995), there were relocation of language classes which accounted for the convenience of users looking for languagerelated materials near literature-related materials. Relocation of architecture in the recent edition of the KDC was also based on user's convenience in looking for all architecture-related materials in one place, not in two separate locations of engineering and arts. In any cases, there are always two distinct cultures interwoven into the system. Thus, the newly introduced concept 'intercultural warrant' is an operational framework for the survey of interactions between distinct cultures.

\subsection{Development of intercultural warrant}

The values of comparative approaches to KOS become evident in considerations of ethics in KO. Ethics in KO is to identify ethical issues caused by the KOS; in other words, $\mathrm{KO}$ ethics address ethical problems from the consequences of constructing, imposing, and use of KOSs. According to Tennis $(2013,42)$, ethical KO "asks the classificationist to be mindful of the choice of terms and relationships between term" concerning marginalized groups or underrepresented groups in the systems. Beghtol (2002) proposed ethical treatment of KOSs in consideration of cultural warrants.

Cultural warrant is to recognize multiple or pluralistic views in organizing knowledge, not limited to a specific time or space, as she also acknowledged the multiplicity of cultural layers. Beyond recognizing pluralistic perspectives in organizing knowledge, a notion of ethical warrants emerges for taking actions to protect a certain view from being suppressed by another. In addition, aiming at either universal or national, library classifications inevitably have multiple cultural influences. In this sense, identification and clarification of those plural views should precede to a protection of a certain view. Thus, a survey of multi (or poly-) cultures embedded in classifications is necessary for taking classification research forward to consideration of ethical warrant.

Despite the KDC's cultural warrant based on the local needs, the adoption of the DDC's main structures in an early developing stage of the KDC must have influenced the cultural warrant of the KDC as well. Furthermore, it is impossible to explain all sociocultural characteristics of a large unit such as one nation, especially regarding international relationships among nations. Thus, the notion of intercultural warrant would shed light on explaining those interwoven sociocultural characteristics. This study is expected to provide theoretical and empirical foundations of the development of 'intercultural warrant' in KO.

\subsection{Visualizing and quantitative-focused analysis of classifications}

The comparative analysis of the KDC and the DDC also examine the cultural warrants embedded in the two systems quantitatively. Analyzing the KDC and the DDC's 
disciplinary distributions discovered major differences between the KDC and the DDC in a macrocosmic view. This disciplinary cultural uniqueness is also expected to imply the KDC's adaptation strategies of the DDC. The different deployment of subdivisions in the main classes of social science and technology from the two classifications potentially provide cases of the adaptive strategies which build the framework of adaptation. In addition, as a compelling methodological approach to KO systems, the visualization techniques with the classification records provides empirical evidence for transitions of knowledge structure, and it also presents potential clues for meanings underneath a body of texts by examining classificatory representations of concepts.

Its goal in comparing the classifications aims at not only identifying differences between the KDC and the DDC but also explaining how they are different through consideration of their different sociocultural contexts and the cross-cultural adoption of a KOS. Comparisons of KOSs, especially those involving research on sociocultural influences, will uncover the role of sociocultural context in KOSs, which appears as differences in knowledge structures. So, the sociocultural differences and the contexts will provide significant research data that can be used to create a framework for future studies

\section{References}

Beall, Julianne. 2003. "Approaches to Expansions: Case Studies from the German and Vietnamese Translations." In World Library and Information Congress: 69th IFLA General Conference and Council, 1-9.

Beghtol, Clare. 2002. "Universal Concepts, Cultural Warrant and Cultural Hospitality." Advances in Knowledge Organization 8: 45-49.

Bliss, Henry Evelyn. 1929. The Organization of Knowledge and the System of the Sciences. New York: HHolt and Company.

Cho, Chansik. 1995. Development of Librarianship in South Korea, 1945-1992: A Historical Study. Rutgers University.

Doyle, Ann M. 2006. "Naming and Reclaiming Indigenous Knowledges in Public Institutions: Intersections of Landscapes and Experience." Advances in Knowledge Organization 10: 435-442.

Fox, Melodie J. 2013. "Rhetorical Space and the Ontogeny of Women in the DDC." Advances in Classification Research Online 23 (1): 59-60.

Hjørland, Birger. 1998. "Theory and Metatheory of Information Science: A New Interpretation." Journal of Documentation 54 (5): 606-21.

Hjørland, Birger. 2008. "What Is Knowledge Organization (KO)?" Knowledge Organization 35 (2/3): 86-101. 
Kim, Yeon-Rye. 2009. "A Comparative Study on the KDC, NDC, and DDC Classification System for Civil Engineering." Journal of the Korean BIBLIA Society for Library and Information Science 20 (3): 219-32.

Kwasnik, Barbara H., and You-Lee Chun. 2004. "Translation of Classifications: Issues and Solutions as Exemplified in the Korean Decimal Classification.” Advances in Knowledge Organization 9: 193-198.

Lee, Hur-Li. 2012. "Epistemic Foundation of Bibliographic Classification in Early China." Journal of Documentation 68 (3): 378-401.

Leidner, Dorothy E. 2010. "Globalization, Culture, and Information: Towards Global Knowledge Transparency.” The Journal of Strategic Information Systems 19 (2): 69-77.

Lester, June, and Wallace C. Koehler. 2007. Fundamentals of Information Studies. New York: Neal-Schuman Publishers.

Mai, Jens-Erik. 2010. "Classification in a Social World: Bias and Trust." Journal of Documentation 66 (5): 627-42.

Neelameghan, A., and K. S. Raghavan. 2012. "Frames of Knowledge: A Perspectivde of Vedic Hinduism and Dravidian Culture." Cultural Frames and Knowledge, Edited by Richard P. Smiraglia \& Hur-Li Lee. Ergon Verlag GMBH, Wurzburg, $19-62$.

Oh, Dong-Geun. 2012. "Developing and Maintaining a National Classification System, Experience from Korean Decimal Classification." Knowledge Organization 39 (2): 72-82.

Oh, Dong-Geun, Yeong-Hwal Bae, and Ji-Suk Yeo. 2008. "Suggestions for the Classes Language and Literature of the 4th Edition of Korean Decimal Classification." Journal of the Korean Society for Library and Information Science 42 (4): 141-57.

Oh, Dong-Geun, Yeong-Hwal Bea, and Ji-Suk Yeo. 2002. KDC 의이해 (Understanding of $K D C$ ).

Oh, Dong-Geun, Yeong-Hwal Bea, and Ji-Suk Yeo. 2009. KDC 의이해 (Understanding of $K D C$ ).

Olson, Hope A. 2001. "The Power to Name: Representation in Library Catalogs." Signs, 639-68.

Olson, Hope A. 2009. "Social Influences on Classification.” In Encyclopedia of Library and Information Sciences, Third Edition, 4806-13. Taylor \& Francis. Salah, Almila Akdag, Cheng Gao, Krzysztof Suchecki, Andrea Scharnhorst, and Richard P. Smiraglia. 2012. "The Evolution of Classification Systems: Ontogeny of the UDC." Advances in Knowledge Organization 13: 51-57. 
Sayers, W. C. Berwick. 1926. A Manual of Classification for Librarians \& Bibliographers, by W. C. Berwick Sayers ... With Illustrations and Bibliography. London, Grafton \& Co.

Smiraglia, Richard. 2014. Cultural Synergy in Information Institutions. Springer.

Smiraglia, Richard, Andrea Scharnhorst, Almila Akdag Salah, and Cheng Gao. 2013.

"UDC in Action." Classification and visualization: interfaces to knowledge.

Tennis, Joseph T. 2006. "Versioning Concept Schemes for Persistent Retrieval."

Bulletin of the American Society for Information Science and Technology 32 (5): 13-16.

Tennis, Joseph T. 2007. "Diachronic and Synchronic Indexing: Modeling Conceptual

Change in Indexing Languages." Proceedings of the Annual Conference of

CAIS/Actes du congrès annuel de l'ACSI.

Tennis, Joseph T. 2012. "The Strange Case of Eugenics: A Subject's Ontogeny in a

Long-Lived Classification Scheme and the Question of Collocative Integrity."

Journal of the American Society for Information Science and Technology 63 (7): $1350-59$.

Tennis, Joseph T. 2013. "Ethos and Ideology of Knowledge Organization: Toward

Precepts for an Engaged Knowledge Organization." Knowledge Organization 40 (1): 42-49.

Xie, Ming. 2011. Conditions of Comparison: Reflections on Comparative

Intercultural Inquiry. Bloomsbury Publishing USA.

Yeo, Ji-suk, Joonman Lee, and Dong-Geun Oh. 2008. "The Improvements of the

Subject Chemical Engineering in the 4th Edition of Korean Decimal

Classification." Journal of Korean Library and Information Science Society 39

(2): 249-66.

Yeo, Ji-Suk, Mi-Sung Park, Myun Hwang, and Dong-Geun Oh. 2008. "KDC 제 4 판

컴퓨터과학분야 전개의 개선방안 (Improvement plan on development of

Computer Science in the KDC 4 edition)." Journal of Korean Library and

Information Science Society 39 (3): 345-68. 